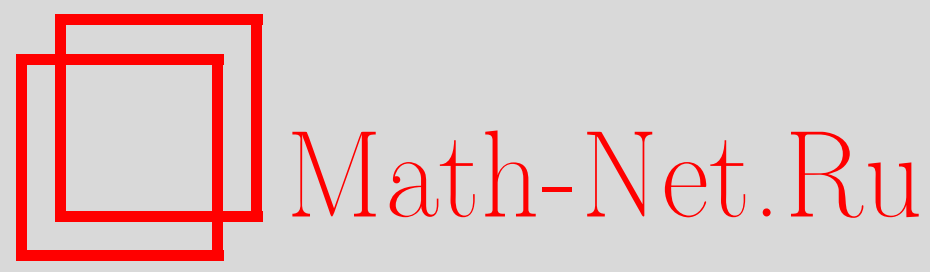

В. Ф. Бутузов, Н. Н. Нефедов, Сингулярно возмущенная краевая задача для уравнения второго порядка в случае смены устойчивости, Матем. заметки, 1998, том 63, выпуск $3,354-362$

DOI: https://doi.org/10.4213/mzm1289

Использование Общероссийского математического портала Math-Net.Ru подразумевает, что вы прочитали и согласны с пользовательским соглашением http://www.mathnet.ru/rus/agreement

Параметры загрузки:

IP: 54.81 .137 .203

26 апреля 2023 г., 17:05:10

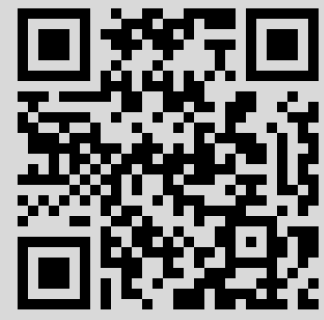




\title{
СИНГУЛЯРНО ВОЗМУЩЕННАЯ КРАЕВАЯ ЗАДАЧА ДЛЯ УРАВНЕНИЯ ВТОРОГО ПОРЯДКА В СЛУЧАЕ СМЕНЫ УСТОЙЧИВОСТИ
}

\author{
В. Ф. Бутузов, Н.Н. Нефёдов
}

Рассмотрена краевая задача для нелинейного сингулярно возмущенного дифференциального уравнения второго порядка в случае смены устойчивости в результате пересечения корней вырожденного уравнения. Методом дифференциальных неравенств доказано существование решения, у которого предельное является негладким.

Библиограффия: 7 названий.

1. Введение. Рассмотрим краевую задачу

$$
\begin{gathered}
L_{\varepsilon} y \equiv \varepsilon^{2} y^{\prime \prime}-f(x, y)=0, \quad 0<x<1, \\
y^{\prime}(0)=y^{\prime}(1)=0,
\end{gathered}
$$

где $\varepsilon>0$ - мальй параметр, $f(x, y)$ - дважды непрерьвно дифференцируемая функция.

Пусть вырожденное уравнение $f(x, y)=0$ имеет корень $y=\bar{y}(x)$ и пусть

$$
\bar{f}_{y}(x) \equiv f_{y}(x, \bar{y}(x))>0 \text { при } 0 \leqslant x \leqslant 1 .
$$

Отметим, что условие (3) обеспечивает изолированность корня $\bar{y}(x)$. При этих условиях имеет место

Теорема 1. При достаточно малых $\varepsilon>0$ существует решение $y(x, \varepsilon)$ задачи (1), (2) такое, что

$$
y(x, \varepsilon)=\bar{y}(x)+O(\varepsilon), \quad x \in[0,1] .
$$

Через $O(\varepsilon)$ здесь и далее обозначаются функции, которые имеют при всех достаточно малых $\varepsilon$ оценку $|O(\varepsilon)| \leqslant C \varepsilon$, где $C$ - не зависящее от $\varepsilon$ положительное число.

Теорема 1 хорошо известна (см., например, [1]). Наиболее просто она доказывается методом дифференциальных неравенств, основанном на теоремах Нагумо (см. [2]). Этот метод базируется на понятиях нижнего и верхнего решения.

Функции $\underline{Y}(x, \varepsilon)$ и $\bar{Y}(x, \varepsilon)$ называются соответственно нижним и верхним решениями задачи (1), (2), если они удовлетворяют следуюшим трем условиям:

$\left.1^{\circ}\right) \underline{Y} \leqslant \bar{Y}$ при $0 \leqslant x \leqslant 1$;

$\left.2^{\circ}\right) L_{\varepsilon} \underline{Y} \geqslant 0, L_{\varepsilon} \bar{Y} \leqslant 0$ при $0<x<1$;

$\left.3^{\circ}\right) \underline{Y}^{\prime}(0, \varepsilon) \geqslant 0 \geqslant \bar{Y}^{\prime}(0, \varepsilon), \underline{Y}^{\prime}(1, \varepsilon) \leqslant 0 \leqslant \bar{Y}^{\prime}(1, \varepsilon)$

(здесь штрих означает производную по $x$ ).

Работа выполнена при частичной финансовой поддержке Российского фонда фундаментальнх исследований, грант № 96-01-00694. 
ДОКАЗАТЕЛЬСТВО ТЕОРЕМЫ 1. Если существуют нижнее и верхнее решения, то существует решение $y(x, \varepsilon)$ задачи $(1),(2)$, удовлетворяющее неравенствам

$$
\underline{Y}(x, \varepsilon) \leqslant y(x, \varepsilon) \leqslant \bar{Y}(x, \varepsilon) \text { при } 0 \leqslant x \leqslant 1 .
$$

Для задачи (1), (2) при условии (3) можно найти нижнее и верхнее решения для достаточно малых $\varepsilon$ в виде

$$
\underline{Y}=\bar{y}(x)-\varepsilon z(x, \varepsilon), \quad \bar{Y}=\bar{y}(x)+\varepsilon z(x, \varepsilon), \quad z(x, \varepsilon)=A+\exp \left\{-\frac{k x}{\varepsilon}\right\}+\exp \left\{-\frac{k(1-x)}{\varepsilon}\right\},
$$

где $A$ и $k$ - достаточно большие положительные числа. В самом деле, условие $1^{\circ}$ ), очевидно, вьполнено. Проверим вьполнение условий $3^{\circ}$ ) и $2^{\circ}$ ). Имеем

$$
\underline{Y}^{\prime}(0, \varepsilon)=\bar{y}^{\prime}(0)+k-k \exp \left\{-\frac{k}{\varepsilon}\right\}
$$

и, следовательно, $\underline{Y}^{\prime}(0, \varepsilon) \geqslant 0$ при достаточно большом $k$ и достаточно мальх $\varepsilon$. Аналогично проверяются остальные неравенства из $3^{\circ}$ ). Перейдем к условию $2^{\circ}$ ):

$$
\begin{aligned}
L_{\varepsilon} \bar{Y}= & \varepsilon^{2} \bar{Y}^{\prime \prime}-f(x, \bar{Y})=\varepsilon^{2} \bar{y}+\varepsilon k^{2}\left(\exp \left\{-\frac{k x}{\varepsilon}\right\}+\exp \left\{-\frac{k(1-x)}{\varepsilon}\right\}\right) \\
& -f(x, \bar{y}(x))-\bar{f}_{y}(x) \varepsilon\left(A+\exp \left\{-\frac{k x}{\varepsilon}\right\}+\exp \left\{-\frac{k(1-x)}{\varepsilon}\right\}\right)+O\left(\varepsilon^{2}\right) \\
= & -\varepsilon\left(\bar{f}_{y}(x) A-\left(k^{2}-\bar{f}_{y}(x)\right)\left(\exp \left\{-\frac{k x}{\varepsilon}\right\}+\exp \left\{-\frac{k(1-x)}{\varepsilon}\right\}\right)\right)+O\left(\varepsilon^{2}\right)
\end{aligned}
$$

Отсюда видно, что в силу (3) неравенство $L_{\varepsilon} \bar{Y} \leqslant 0$ выполнено при достаточно большом $A$ и достаточно малых $\varepsilon$ за счет слагаемого $-\varepsilon \bar{f}_{y}(x) A$. Аналогично проверяется условие $L_{\varepsilon} \underline{Y} \geqslant 0$.

Итак, функции (6) являются нижним и верхним решениями задачи $(1),(2)$; поэтому существует решение $y(x, \varepsilon)$ этой задачи, удовлетворяющее неравенствам (5), откуда следует, что $y(x, \varepsilon)=\bar{y}(x)+O(\varepsilon)$, и теорема 1 доказана.

Сделаем несколько замечаний.

ЗАмЕчАнИЕ 1. Нетрудно получить более точное чем (4) асимптотическое представление для решения $y(x, \varepsilon)$. А именно, вне сколь угодно малых, но не зависящих от $\varepsilon$, $\delta$-окрестностей граничньх точек $x=0$ и $x=1$ имеет место равенство

$$
y(x, \varepsilon)=\bar{y}(x)+O\left(\varepsilon^{2}\right)
$$

Чтобы доказать это, нужно видоизменить нижнее и верхнее решения следуюшим образом. Вместо слагаемого $A \varepsilon$ нужно ввести гладкую функцию, которая равна $A \varepsilon$ в $(\delta / 2)$-окрестностях точек $x=0$ и $x=1$, равна $A \varepsilon^{2}$ вне $\delta$-окрестностей этих точек, и изменяется монотонно от $A \varepsilon$ до $A \varepsilon^{2}$ на отрезках $[\delta / 2, \delta]$ и $[1-\delta, 1-\delta / 2]$. 
ЗАмЕчАниЕ 2. Мы рассмотрели случай краевых условий (2). Можно брать и другие краевые условия, например, $y(0)=y(1)=0$. В этом случае с помощю метода дифференциальных неравенств можно доказать существование решения $y(x, \varepsilon)$, имеющего асимптотическое представление

$$
y(x, \varepsilon)=\bar{y}(x)+\Pi_{0}\left(\frac{x}{\varepsilon}\right)+\Pi_{1}\left(\frac{1-x}{\varepsilon}\right)+O(\varepsilon),
$$

где $\Pi_{0}$ и $\Pi_{1}-$ пограничные функции. Построение нижнего и верхнего решения в этом случае несколько усложняется (см., например, [3]).

ЗАмЕчАниЕ 3. Приведенное доказательство теоремы 1 существенно опирается на условие (3); это условие обеспечивает вьполнение неравенств $L_{\varepsilon} \underline{Y} \geqslant 0, L_{\varepsilon} \bar{Y} \leqslant 0$.

ЗАмечание 4. Решение задачи (1), (2), вообще говоря, не единственно. Вырожденное уравнение может иметь несколько корней, удовлетворяющих условию (3), и тогда для каждого такого корня существует решение задачи (1), (2), удовлетворяющее условию (4). Кроме того, наряду с решениями, удовлетворяющими условию (4), задача $(1),(2)$ может иметь решение с асимптотикой вида (8), т.е. с пограничньми слоями в окрестностях точек $x=0$ и $x=1$, а также решение с внутренними переходньпии слоями (контрастные структуры) типа ступеньки или всплеска [1]. Существование таких решений и построение их асимптотик существенно опирается, как и в рассмотренном случае, на условие (3). Подчеркнем, что неравенство $\bar{f}_{y}(x)>0$ должно вьполнятся во всех точках отрезка $0 \leqslant x \leqslant 1$.

2. Постановка задачи. Целью данной работы является рассмотрение случая, когда в некоторой точке нарушается условие (3), что связано с пересечением корней вырожденного уравнения.

I. Пусть вырожденное уравнение $f(x, y)=0$ имеет два корня $y=y_{1}(x)$ и $y=y_{2}(x)$, причем существует $x_{0} \in(0,1)$ такое, что

a) $y_{1}(x)>y_{2}(x)$ при $0 \leqslant x<x_{0}, y_{1}\left(x_{0}\right)=y_{2}\left(x_{0}\right), y_{1}(x)<y_{2}(x)$ при $x_{0}<x \leqslant 1$;

б) $y_{2}^{\prime}\left(x_{0}\right)>y_{1}^{\prime}\left(x_{0}\right)$.

Условие I показывает, что в точке $x_{0}$ корни пересекаются. Поэтому

$$
f_{y}\left(x_{0}, y_{1}\left(x_{0}\right)\right)=f_{y}\left(x_{0}, y_{2}\left(x_{0}\right)\right)=0 .
$$

II. Пусть

$$
\begin{aligned}
& f_{y}\left(x, y_{1}(x)\right)>0 \text { при } 0 \leqslant x<x_{0}, \quad f_{y}\left(x, y_{1}(x)\right)<0 \text { при } x_{0}<x \leqslant 1 \text {, } \\
& f_{y}\left(x, y_{2}(x)\right)<0 \text { при } 0 \leqslant x<x_{0}, \quad f_{y}\left(x, y_{2}(x)\right)>0 \text { при } x_{0}<x \leqslant 1 \text {. }
\end{aligned}
$$

Условие II показьвает, что в точке $x_{0}$ на каждом из корней $y_{1}(x)$ и $y_{2}(x)$ производная $f_{y}$ меняет знак, т.е. в точке $x_{0}$ меняется тип устойчивости корней вырожденного уравнения. Поэтому такой случай мы называем случаем смены устойчивости.

Образуем функцию

$$
\widehat{y}(x)= \begin{cases}y_{1}(x), & 0 \leqslant x \leqslant x_{0}, \\ y_{2}(x), & x_{0} \leqslant x \leqslant 1 .\end{cases}
$$

В силу Іб) эта функция имеет в точке $x_{0}$ разрьв (скачок) первой производной, причем $\widehat{y}^{\prime}\left(x_{0}+0\right)-\widehat{y}^{\prime}\left(x_{0}-0\right)>0$. В силу II $\widehat{f}_{y}(x) \equiv f_{y}(x, \widehat{y}(x))>0$ при $x \neq x_{0}$, а в силу (9) $\widehat{f}_{y}\left(x_{0}\right)=0$, т.е. в точке $x_{0}$ нарушается условие $(3)$. 
Поставим вопрос: существует ли решение $y(x, \varepsilon)$ задачи $(1),(2)$ такое, что

$$
y(x, \varepsilon)=\widehat{y}(x)+O\left(\varepsilon^{\beta}\right), \beta>0 ?
$$

Положительный ответ на этот вопрос будет получен с помощью метода дифференциальных неравенств при еще двух условиях.

III. $\widehat{f}_{y y}\left(x_{0}\right) \equiv f_{y y}\left(x_{0}, \widehat{y}\left(x_{0}\right)\right)>0$.

IV. $\widehat{f}_{y}(x) \equiv f_{y}\left(x, \widehat{y}_{0}(x)\right) \geqslant M\left|x-x_{0}\right|$, где $M$ - положительное число.

Простейшим примером функции $f(x, y)$, которая удовлетворяет условиям II-IV, , яBляется квадратичная относительно переменной $y$ функция $f(x, y)=\left(y-y_{1}(x)\right)\left(y-y_{2}(x)\right)$ в случае, когда корни $y_{1}(x)$ и $y_{2}(x)$ удовлетворяют условию I.

Отметим, что начальная задача для сингулярно возмущенных уравнений в случае смены устойчивости рассматривалась в [4] и [5]. Использовавшийся в [4] метод специфичен именно для начальных задач и не пригоден для краевой задачи. В [5] использован метод дифференциальных неравенств Чапльгина. При этом требовалось, чтобы $\widehat{y}(x)$ было нижним решением, а процедура построения верхнего решения такова, что к краевьм задачам она не применима.

3. Построение верхнего решения. Прежде всего заметим, что построить нижнее и верхнее решения в таком же виде, как при доказательстве теоремы 1 , т.е. в виде

$$
\begin{gathered}
\underline{Y}=\widehat{y}(x)-z(x, \varepsilon), \bar{Y}=\widehat{y}(x)+z(x, \varepsilon) \\
z(x, \varepsilon)=A \varepsilon^{\beta}+\varepsilon\left(\exp \left\{-\frac{k x}{\varepsilon}\right\}+\exp \left\{-\frac{k(1-x)}{\varepsilon}\right\}\right),
\end{gathered}
$$

где $\beta>0$, невозможно по двум причинам.

Во-первых, функция $\widehat{y}(x)$ имеет разрыв производной в точке $x_{0}$, причем $\widehat{y}^{\prime}\left(x_{0}+0\right)-$ $\widehat{y}^{\prime}\left(x_{0}-0\right)>0$ в силу Іб). Такой (положительньй) скачок производной в отдельных точках допустим для нижнего решения, но не допустим для верхнего решения (см. [6]). Во-вторых, поскольку $\widehat{f}_{y}\left(x_{0}\right)=0$, а $\widehat{f}_{y y}\left(x_{0}\right)>0$, вблизи точки $x_{0}$ указанная функция $\underline{Y}$ не будет, вообще говоря, удовлетворять требуемому неравенству $L_{\varepsilon} \underline{Y} \geqslant 0$.

Таким образом, построение нижнего и верхнего решений в случае смены устойчивости является более сложной проблемой.

Мы построим сначала верхнее решение в некоторой $\delta$-окрестности точки $x_{0}$, где $\delta$ достаточно мало, но не зависит от $\varepsilon$, а затем укажем, как продолжить это решение на весь отрезок $[0,1]$. С этой целью сгладим функцию $\widehat{y}(x)$ с помощью известной процедуры, которая применялась ранее в задачах с негладкими членами асимптотики (см., например, [7]). Введем переменную

$$
\xi=\frac{x-x_{0}}{x^{\alpha}}
$$

где $\alpha$ - положительное число, которое будет выбрано ниже. Положим

$$
\omega(\xi)=\frac{1}{\sqrt{\pi}} \int_{-\infty}^{\xi} \exp \left\{-s^{2}\right\} d s,
$$

и вместо $\widehat{y}(x)$ введем всюду гладкую функцию

$$
\widetilde{y}(x)=y_{1}(x) \omega(-\xi)+y_{2}(x) \omega(\xi) .
$$


Используя равенство $\omega(-\xi)+\omega(\xi)=1$, получаем при $x \leqslant x_{0}$ (т.е. при $\left.\xi \leqslant 0\right)$

$$
\widetilde{y}(x)=y_{1}(x)+\left(y_{2}(x)-y_{1}(x)\right) \omega(\xi)=y_{1}(x)+O\left(x-x_{0}\right) \omega(\xi)=y_{1}(x)+O\left(\varepsilon^{\alpha}\right) .
$$

Аналогично, при $x \geqslant x_{0}$ имеем $\widetilde{y}(x)=y_{2}(x)+O\left(\varepsilon^{\alpha}\right)$. Таким образом, $\widetilde{y}(x)=\widehat{y}(x)+O\left(\varepsilon^{\alpha}\right)$. Нетрудно заметить, что $\widetilde{y}(x) \leqslant \widehat{y}(x)$, т.е. процедура сглаживания дает кривую $\widetilde{y}(x)$, расположенную ниже $\widehat{y}(x)$.

В $\delta$-окрестности точки $x_{0}$ верхнее решение будем искать в виде

$$
\bar{Y}=\widetilde{y}(x)+A \varepsilon^{\beta}
$$

где $0<\beta \leqslant \alpha, A>0$. При достаточно большом $A$ получаем неравенство $\bar{Y}>\widehat{y}(x)$.

Покажем, что можно выбрать $\alpha, \beta$ и $A$ так, что в $\delta$-окрестности точки $x_{0}$ будет выполнено условие $L_{\varepsilon} \bar{Y} \leqslant 0$. Имеем

$$
\begin{aligned}
L_{\varepsilon} \bar{Y} \equiv & \varepsilon^{2} \bar{Y}-f(x, \bar{Y}) \\
= & \varepsilon^{2}\left(y_{1}^{\prime \prime}(x) \omega(-\xi)+y_{2}^{\prime \prime}(x) \omega(\xi)\right)+\varepsilon^{2-\alpha} \frac{2}{\sqrt{\pi}}\left(y_{2}^{\prime}(x)-y_{1}^{\prime}(x)\right) \exp \left\{-\xi^{2}\right\} \\
& +\varepsilon^{2-2 \alpha} \frac{1}{\sqrt{\pi}}\left(y_{1}(x)-y_{2}(x)\right) \xi \exp \left\{-\xi^{2}\right\} \\
& -\left(f\left(x, \widehat{y}_{0}(x)\right)+\widehat{f}_{y}(x)\left(A \varepsilon^{\beta}+O\left(\varepsilon^{\alpha}\right)\right)+\frac{1}{2} \widehat{f}_{y y}(x)\left(A \varepsilon^{\beta}+O\left(\varepsilon^{\alpha}\right)\right)^{2}+o\left(\varepsilon^{2 \beta}\right)\right) \\
= & O\left(\varepsilon^{2-\alpha}\right)-\widehat{f}_{y}(x)\left(A \varepsilon^{\beta}+O\left(\varepsilon^{\alpha}\right)\right)-\frac{1}{2} \widehat{f}_{y y}(x)\left(A \varepsilon^{\beta}+O\left(\varepsilon^{\alpha}\right)\right)^{2}+o\left(\varepsilon^{2 \beta}\right) .
\end{aligned}
$$

Первое слагаемое в правой части равенства (величина $\left.O\left(\varepsilon^{2-\alpha}\right)\right)$ может иметь любой знак. Второе слагаемое обращается в 0 в точке $x_{0}$ и отрицательно в остальных точках. Третье слагаемое будет отрицательным всюду в $\delta$-окрестности точки $x_{0}$, если величину $\delta$ взять столь малой, чтобы в $\delta$-окрестности точки $x_{0}$ выполнялось неравенство $\widehat{f}_{y y}(x)>0$. Это возможно в силу условия III. При этом третье слагаемое является величиной порядка $A^{2} \varepsilon^{2 \beta}$. Поэтому если $2 \beta \leqslant 2-\alpha$, то при достаточно большом $A$ и достаточно малом $\varepsilon$ третье слагаемое будет доминирующим и обеспечит выполнение неравенства $L_{\varepsilon} \bar{Y} \leqslant 0$ в $\delta$-окрестности точки $x_{0}$.

Таким образом, $\alpha$ и $\beta$ нужно взять удовлетворяющими неравенствам $0<\beta \leqslant \alpha$, $2 \beta \leqslant 2-\alpha$. Отсюда получаем $0<\beta \leqslant 2 / 3, \beta \leqslant \alpha \leqslant 2-2 \beta$. Наилучший выбор $\alpha$ и $\beta$, минимизирующий отклонение $\bar{Y}$ от $\widehat{y}(x)$, таков: $\alpha=\beta=2 / 3$.

Итак, в $\delta$-окрестности точки $x_{0}$ построеноверхнее решение в виде $\bar{Y}=\widehat{y}(x)+O\left(\varepsilon^{2 / 3}\right)$.

Вне $\delta$-окрестности точки $x_{0}$ вьполняется неравенство $\widehat{f}_{y}(x) \geqslant C>0$, и поэтому здесь можно взять верхнее решение в том же виде, как и при доказательстве теоремы 1 , т.е. в виде $(6)$ с заменой $\bar{y}(x)$ на $\widehat{y}(x)$. Чтобы построить всюду гладкое верхнее решение, можно поступить так: в $(\delta / 2)$-окрестности точки $x_{0}$ верхнее решение берется в виде $(11)$, вне $\delta$-окрестности - в виде (6), а на отрезках $\delta / 2 \leqslant\left|x-x_{0}\right| \leqslant \delta$ осуществляется гладкий монотонньй переход от одного вида верхнего решения к другому. При этом неравенство $L_{\varepsilon} \bar{Y} \leqslant 0$ будет вьполнено и на этих отрезках, поскольку вне $(\delta / 2)$-окрестности точки $x_{0}$ первое слагаемое в правой части (12) является величиной порядка $O\left(\varepsilon^{2}\right)$, а не $O\left(\varepsilon^{2-\alpha}\right)$. Кроме того, $\bar{Y}$ будет удовлетворять условию $\left.3^{\circ}\right)$.

Итак, верхнее решение построено. 
4. Построение нижнего решения. В качестве главного члена нижнего решения можно взять $\widehat{y}(x)$, не сглаживая его, поскольку для нижнего решения допустим положительный скачок производной, какой имеет $\widehat{y}(x)$ в точке $x_{0}$. Однако, построить нижнее решение в виде $\underline{Y}=\widehat{y}(x)-A \varepsilon^{\beta}$ не удается. В самом деле, при таком выборе $\underline{Y}$ получаем

$$
L_{\varepsilon} \underline{Y}=O\left(\varepsilon^{2}\right)+\widehat{f}_{y}(x) A \varepsilon^{\beta}-\frac{1}{2} \widehat{f}_{y y}(x) A^{2} \varepsilon^{2 \beta}+o\left(\varepsilon^{2 \beta}\right) .
$$

Во всех точках (кроме $x_{0}$, где $\widehat{y}(x)$ недифференцируема) должно вьполняться неравенство $L_{\varepsilon} \underline{Y} \geqslant 0$. Но так как $\widehat{f}_{y}\left(x_{0}\right)=0$, вблизи точки $x_{0}$ доминируюшим является третье слагаемое, а оно в силу III отрицательно.

Всюду, за исключением малых окрестностей граничных точек $x=0$ и $x=1$, удается построить нижнее решение в виде

$$
\underline{Y}=\widehat{y}-A \varepsilon^{\beta}+B \varepsilon^{2 \alpha} \xi^{2} \exp \{-|\xi|\}, \quad \xi=\frac{x-x_{0}}{\varepsilon^{\alpha}}
$$

где $A$ и $B$ - достаточно большие положительные числа, причем $B<A ; 0<\beta \leqslant 2 \alpha$, более точньй выбор $\alpha$ и $\beta$ сделан ниже. Ясно, что при $B<A$ имеем неравенство $\underline{Y}<\widehat{y}(x)$. Далее,

$$
\begin{aligned}
L_{\varepsilon} \underline{Y}= & \varepsilon^{2}\left(\widehat{y}_{0}^{\prime \prime}(x)+B\left(2-4|\xi|+\xi^{2}\right) \exp \{-|\xi|\}\right)-\widehat{f}_{y}(x)\left(B \varepsilon^{2 \alpha} \xi^{2} \exp \{-|\xi|\}-A \varepsilon^{\beta}\right) \\
& -\frac{1}{2} \widehat{f}_{y y}(x)\left(B \varepsilon^{2 \alpha} \xi^{2} \exp \{-|\xi|\}-A \varepsilon^{\beta}\right)^{2}+o\left(\varepsilon^{2 \beta}\right) .
\end{aligned}
$$

Покажем, что можно так выбрать $B, A, \alpha, \beta$, что при $0<x<1\left(x \neq x_{0}\right)$ будет вьполнено неравенство $L_{\varepsilon} \underline{Y} \geqslant 0$.

Рассмотрим сначала промежутки $0<\left|x-x_{0}\right| \leqslant k \varepsilon^{\alpha}$ (т.е. $0<|\xi| \leqslant k$ ), причем возьмем $k$ столь мальм, что $2-4|\xi|+\xi^{2}>0$ при $|\xi| \leqslant k$. Если $\beta>1$, то два последних слагаемых в правой части (14) являются величинами порядка $o\left(\varepsilon^{2}\right)$. Второе слагаемое положительно, но оно сколь угодно мало вблизи точки $x_{0}\left(\right.$ так как $\widehat{f}_{y}\left(x_{0}\right)=0$ ) и потому не является главным вблизи $x_{0}$. Первое же слагаемое является величиной порядка $\varepsilon^{2}$ и поэтому при достаточно большом $B$ и достаточно малых $\varepsilon$ обеспечивает вьполнение неравенства $L_{\varepsilon} \underline{Y} \geqslant 0$ на промежутках $0<\left|x-x_{0}\right| \leqslant k \varepsilon^{\alpha}$. Таким образом, последнее слагаемое в выражении (13) для $\underline{Y}$ играет определяющую роль в малой (порядка $\varepsilon^{\alpha}$ ) окрестности точки $x_{0}$.

Вне этой окрестности точки $x_{0}$, т.е. при $\left|x-x_{0}\right|>k \varepsilon^{\alpha}$, доминирующим станет второе слагаемое в правой части (14). В самом деле, в силу условия IV при $\left|x-x_{0}\right|>k \varepsilon^{\alpha}$ имеем

$$
\widehat{f}_{y}(x) \geqslant M\left|x-x_{0}\right|>M k \varepsilon^{\alpha}
$$

и, следовательно, для второго слагаемого в правой части (14) получаем оценку

$$
-\widehat{f}_{y}(x)\left(-A \varepsilon^{\beta}+B \varepsilon^{2 \alpha} \xi^{2} \exp \{-|\xi|\}\right) \geqslant M k(A-B) \varepsilon^{\alpha+\beta} .
$$

Если $\alpha+\beta \leqslant 2$, то при достаточно большом $A$ и достаточно мальх $\varepsilon$ второе слагаемое становится доминирующим и обеспечивает вьполнение неравенства $L_{\varepsilon} \underline{Y} \geqslant 0$ всюду при $\left|x-x_{0}\right|>k \varepsilon^{\alpha}$.

Таким образом, чтобы построить нижнее решение в виде $(13)$, нужно взять $\alpha$ и $\beta$, удовлетворяющие неравенствам $0<\beta \leqslant 2 \alpha, \beta>1, \alpha+\beta \leqslant 2$. Отсюда получаем 
$1<\beta \leqslant 4 / 3, \beta / 2 \leqslant \alpha \leqslant 2-\beta$. Наилучший выбор $\alpha$ и $\beta$, обеспечивающий наименьшее отклонение $\underline{Y}$ от $\widehat{y}(x)$, таков: $\beta=2 \alpha=4 / 3$. При этом выборе $\alpha$ и $\beta$ имеем $\underline{Y}=\widehat{y}(x)-O\left(\varepsilon^{4 / 3}\right)$. Заметим, что такое $\underline{Y}$ не удовлетворяет, вообще говоря, условию $3^{\circ}$ ). Поэтому в сколь угодно малых, но не зависящих от $\varepsilon, \delta$-окрестностях граничных точек $x=0$ и $x=1$ возьмем $\underline{Y}$ в виде (6), вне $\delta$-окрестностей этих точек в виде $(13)$, а на отрезках $[\delta, 2 \delta]$ и $[1-2 \delta, 1-\delta]$ произведем гладкое сопряжение этих функций. При этом неравенство $L_{\varepsilon} \underline{Y} \geqslant 0$ будет вьполнено всюду и, кроме того, $\underline{Y}$ будет удовлетворять условию $\left.3^{\circ}\right)$.

Так как $\underline{Y}<\widehat{y}(x)$, а $\bar{Y}>\widehat{y}(x)$, то $\underline{Y}<\bar{Y}$, т.е. выполнено условие $\left.1^{\circ}\right)$. Таким образом, построенные функции $\underline{Y}$ и $\bar{Y}$ являются нижним и верхним решениями задачи $(1),(2)$.

5. Основной результат. Построенное верхнее решение $\bar{Y}$ отличается от $\widehat{y}(x)$ на величину порядка $\varepsilon$ всюду на отрезке $[0,1]$, за исключением малой $\delta$-окрестности точки $x_{0}$, где разность $\bar{Y}-\widehat{y}(x)$ является величиной порядка $\varepsilon^{2 / 3}$. Нижнее решение $\underline{Y}$ отличается от $\widehat{y}(x)$ на величину порядка $\varepsilon^{4 / 3}$ всюду, кроме малых окрестностей точек $x=0$ и $x=1$, где разность $\widehat{y}-\underline{Y}$ является величиной порядка $\varepsilon$. Отсюда вытекает следующее утверждение.

ТЕОРема 2. Если выполнены условия I-IV, то при достаточно малых $\varepsilon>0$ существует решение $y(x, \varepsilon)$ задачи (1), (2) такое, что $y(x, \varepsilon)=\widehat{y}(x)+O\left(\varepsilon^{2 / 3}\right)$ в $\delta$-окрестности точки $x_{0}$ и $y(x, \varepsilon)=\widehat{y}(x)+O(\varepsilon)$ в остальной части отрезка $[0,1]$.

Воспользовавшись замечанием 1 , можно написать более точное асимптотическое представление $(7)$ для $y(x, \varepsilon)$ вне $\delta$-окрестностей точек $x=x_{0}, x=0$ и $x=1: y(x, \varepsilon)=$ $\widehat{y}(x)+O\left(\varepsilon^{2}\right)$.

6. Случай зависимости функции $f$ от $\varepsilon$. Выше рассмотрен случай, когда функция $f$ не зависит от $\varepsilon$. Если же $f=f(x, y, \varepsilon)$ и функция $f(x, y, 0)$ удовлетворяет условиям I-IV, то существенную роль играет знак производной $\widehat{f}_{\varepsilon}$ в точке $x_{0}$. Рассмотрим два случая.

1) $\widehat{f}_{\varepsilon}\left(x_{0}\right) \equiv f_{\varepsilon}\left(x_{0}, \widehat{y}\left(x_{0}\right), 0\right)<0$.

В этом случае в $\delta$-окрестности точки $x_{0}$ можно построить верхнее решение в виде

$$
\bar{Y}=\widetilde{y}(x)+A \sqrt{\varepsilon}=\widehat{y}(x)+O(\sqrt{\varepsilon})+A \sqrt{\varepsilon},
$$

где $A$ - достаточно большое число, а $\widetilde{y}(x)$ определяется формулой $(10)$, причем $\xi=$ $\left(x-x_{0}\right) / \sqrt{\varepsilon}$. Действительно, вместо равенства (12) имеем

$$
L_{\varepsilon} \bar{Y}=O\left(\varepsilon^{3 / 2}\right)-\widehat{f}_{y}(x)(A \sqrt{\varepsilon}+O(\sqrt{\varepsilon}))-\widehat{f}_{\varepsilon}(x) \varepsilon-\frac{1}{2} \widehat{f}_{y y}(x)(A \sqrt{\varepsilon}+O(\sqrt{\varepsilon}))^{2},
$$

откуда видно, что при достаточно большом $A$ и достаточно малых $\varepsilon$ в $\delta$-окрестности точки $x_{0}$ (где $\widehat{f}_{y y}(x)>0$ ) неравенство $L_{\varepsilon} \bar{Y} \leqslant 0$ будет вьполнено за счет члена $\frac{1}{2} \widehat{f}_{y y}(x) A^{2} \varepsilon$.

Вне $\delta$-окрестности точки $x_{0}$ верхнее решение можно построить в виде $(6)$ с заменой $\bar{y}(x)$ на $\widehat{y}(x)$.

Нижнее решение $\underline{Y}$ на всем отрезке $[0,1]$ можно взять также в виде $(6)$ с заменой $\bar{y}(x)$ на $\widehat{y}(x)$. При этом в $\delta$-окрестности точки $x_{0}$ (где $\widehat{f_{\varepsilon}}(x)<0$ ) неравенство $L_{\varepsilon} \underline{Y} \geqslant 0$ будет вьполнено при достаточно малых $\varepsilon$ за счет члена $-\widehat{f}_{\varepsilon}(x) \varepsilon$ (см. $\left.(15)\right)$, а вне этой $\delta$-окрестности - за счет члена $\widehat{f}_{y}(x) A \varepsilon$. Заметим, что если $\widehat{f}_{\varepsilon}(x)<0$ на всем отрезке $[0,1]$, то в качестве нижнего решения можно взять $\widehat{y}(x)$.

Указанные нижнее и верхнее решения позволяют сформулировать следующую теорему. 
Tеорема 3. Если $f=f(x, y, \varepsilon)$, функиия $f(x, y, 0)$ удовлетворяет условиям I-IV $и \widehat{f}_{\varepsilon}\left(x_{0}\right)<0$, то при достаточно малых $\varepsilon$ существует решение $y(x, \varepsilon)$ задачи (1), (2) такое, что $y(x, \varepsilon)=\widehat{y}(x)+O(\sqrt{\varepsilon})$ в $\delta$-окрестности точки $x_{0} u$ $y(x, \varepsilon)=\widehat{y}(x)+O(\varepsilon)$ в остальной части отрезка $[0,1]$.

Отметим, что в рассмотренном случае в отличие от случая $f=f(x, y)$ вне $\delta$-окрестностей точек $x=x_{0}, x=0$ и $x=1$ равенство $y(x, \varepsilon)=\widehat{y}(x)+O\left(\varepsilon^{2}\right)$ уже не имеет места. Чтобы получить более точную асимптотику $y(x, \varepsilon)$, нужно добавить следуюший член разложения, которьй в данном случае можно определить как решение квадратного уравнения

$$
\frac{1}{2} \widehat{f}_{y y}(x) y_{1}^{2}+\widehat{f}_{y}(x) y_{1}+\widehat{f}_{\varepsilon}(x) \varepsilon=0,
$$

причем нужно взять корень

$$
y_{1}(x, \varepsilon)=-2 \varepsilon \widehat{f}_{\varepsilon}(x)\left(\widehat{f}_{y}(x)+\sqrt{\widehat{f}_{y}^{2}(x)-2 \widehat{f}_{y y}(x) \widehat{f}_{\varepsilon}(x) \varepsilon}\right)^{-1} .
$$

Нетрудно заметить, что $y_{1}(x, \varepsilon)=O(\sqrt{\varepsilon})$ в малой $\delta$-окрестности точки $x_{0}$ и $y_{1}(x, \varepsilon)=$ $O(\varepsilon)$ вне $\delta$-окрестности точки $x_{0}$. Справедлива следуюшая теорема.

ТЕорема 4. Если выполнены условия теоремы 3 , то при достаточно малых $\varepsilon$ существует решение $y(x, \varepsilon)$ задачи (1), (2) такое, что

$$
y(x, \varepsilon)= \begin{cases}\widehat{y}(x)+y_{1}(x, \varepsilon)+O\left(\varepsilon^{3 / 4}\right) & \text { в } \delta \text {-окрестности точки } x_{0}, \\ \widehat{y}(x)+O(\varepsilon) & \text { в } \delta \text {-окрестностях точек } x=0 \text { и } x=1, \\ \widehat{y}(x)+y_{1}(x, \varepsilon)+O\left(\varepsilon^{2}\right) & \text { на отрезках }\left[\delta, x_{0}-\delta\right] u\left[x_{0}+\delta, 1-\delta\right] .\end{cases}
$$

Для доказательства этой теоремы можно построить соответствующие нижнее и верхнее решения, причем в $\delta$-окрестности точки $x_{0}$ удается построить их в виде

$$
\underline{Y}=\widehat{y}(x)+y_{1}(x, \varepsilon)-A \varepsilon^{3 / 4}, \quad \bar{Y}=\widetilde{y}(x)+\widetilde{y}_{1}(x, \varepsilon)+A \varepsilon^{3 / 4},
$$

где $A$ - достаточно большое число, $\widetilde{y}$ и $\widetilde{y}_{1}$ получаются из $\widehat{y}$ и $y_{1}$ по формуле $(10)$, причем $\xi=\left(x-x_{0}\right) / \varepsilon^{3 / 4}$.

2) $\widehat{f}_{\varepsilon}\left(x_{0}\right)>0$.

В этом случае может не сушествовать решения $y(x, \varepsilon)$ задачи $(1),(2)$ такого, что

$$
y(x, \varepsilon)=\widehat{y}(x)+O\left(\varepsilon^{\beta}\right), \quad \beta>0 .
$$

ПримеР. Рассмотрим краевую задачу

$$
\begin{gathered}
\varepsilon^{2} y^{\prime \prime}=y\left(y-x+\frac{1}{2}\right)+\varepsilon, \quad 0<x<1, \\
y^{\prime}(0)=y^{\prime}(1)=0 .
\end{gathered}
$$

Нетрудно убедиться в том, что $f(x, y, 0)=y(y-x+1 / 2)$ удовлетворяет всем условиям I-IV. В частности, уравнение $f(x, y, 0)=0$ имеет два корня $y_{1}=0$ и $y_{2}=x-1 / 2$, пересекаюшиеся в точке $x_{0}=1 / 2$. В данном примере

$$
\widehat{y}(x)= \begin{cases}0, & 0 \leqslant x \leqslant \frac{1}{2} \\ x-\frac{1}{2}, & \frac{1}{2} \leqslant x \leqslant 1\end{cases}
$$


а производная $f_{\varepsilon}=1>0$.

Пусть $y(x)$ - решение задачи (17) (зависимость $y$ от $\varepsilon$ для краткости не указываем). Рассмотрим отрезок $I=\{x: 1 / 2-\sqrt{\varepsilon} \leqslant x \leqslant 1 / 2+\sqrt{\varepsilon}\}$. На этом отрезке

$$
f(x, y, \varepsilon)=\left(y-\frac{1}{2}\left(x-\frac{1}{2}\right)\right)^{2}-\frac{1}{4}\left(x-\frac{1}{2}\right)^{2}+\varepsilon \geqslant \frac{3}{4} \varepsilon
$$

и, следовательно, $y^{\prime \prime}(x)=\varepsilon^{-2} f \geqslant 3 \varepsilon^{-1} / 4$. Поэтому $y^{\prime}(x)$ возрастает на отрезке $I$ и, значит, либо сохраняет знак, либо меняет его в какой-то одной точке отрезка. Допустим, что $y^{\prime}(x)$ обрашается в 0 в точке $x_{1}$. Пусть $x_{1} \leqslant 1 / 2$. Рассмотрим отрезок $\left[x_{1}, 1 / 2+\sqrt{\varepsilon} / 2\right]$ (если $x_{1}>1 / 2$, то нужно рассмотреть отрезок $\left.\left[1 / 2-\sqrt{\varepsilon} / 2, x_{1}\right]\right)$. Применяя формулу конечных приращений, получаем

$$
y^{\prime}\left(\frac{1}{2}+\frac{\sqrt{\varepsilon}}{2}\right)-y^{\prime}\left(x_{1}\right)=y^{\prime \prime}(\theta)\left(\frac{1}{2}+\frac{\sqrt{\varepsilon}}{2}-x_{1}\right) \geqslant \frac{3}{4} \varepsilon^{-1} \frac{\sqrt{\varepsilon}}{2},
$$

откуда $y^{\prime}(1 / 2+\sqrt{\varepsilon} / 2) \geqslant 3 \varepsilon^{-1 / 2} / 8$. Отсюда следует, что $y^{\prime}(x) \geqslant 3 \varepsilon^{-1 / 2} / 8$ при $1 / 2+$ $\sqrt{\varepsilon} / 2 \leqslant x \leqslant 1 / 2+\sqrt{\varepsilon}$. Поэтому

$$
y\left(\frac{1}{2}+\sqrt{\varepsilon}\right)-y\left(\frac{1}{2}+\frac{\sqrt{\varepsilon}}{2}\right)=y^{\prime}\left(\theta_{1}\right) \frac{\sqrt{\varepsilon}}{2} \geqslant \frac{3}{16} .
$$

Мы получили, что на отрезке $[1 / 2+\sqrt{\varepsilon} / 2,1 / 2+\sqrt{\varepsilon}]$ решение $y(x)$ изменяется на конечную величину, бо́льшую $3 / 16$. В то же время $\widehat{y}(x)$ изменяется на этом отрезке на величину $\sqrt{\varepsilon} / 2$. Следовательно, на этом отрезке не может быть вьполнено равенство $y(x)-\widehat{y}(x)=O\left(\varepsilon^{\beta}\right)$, где $\beta>0$. Таким же образом можно доказать, что последнее равенство не имеет места, если $y^{\prime}(x)$ сохраняет свой знак на отрезке $I$. Итак, задача $(17)$ не имеет решения $y(x, \varepsilon)$, удовлетворяющего условию $(16)$.

\section{СПИСОК ЦИТИРОВАННОЙ ЛИТЕРАТУРЫ}

[1] Васильева А. Б., Бутузов В. Ф. Асимптотические методы в теории сингулярных возмущений. М.: Высшая школа, 1990.

[2] Гудков В. В., Клоков Ю. А., Лепин А. Я., Пономарёв В. Д. Двухточечные краевые задачи для обыкновенных дифференциальных уравнений. Рига: Зинатне, 1973.

[3] Нефёдов Н.Н. Метод дифференциальных неравенств для некоторых сингулярно возмущенных задач в частных производных // Дифференц. уравнения. 1993. Т. 31. № 4. С. 719-722.

[4] Lebowitz N. R., Shaar R. J. Exchange of stabilities in autonomous systems // Stud. Appl. Math. 1975. V. 54. № 3. P. 229-259.

[5] Nefedov N., Schneider K., Schuppert A. Jumping Behavior in Singularly Perturbed Systems Modelling Bimolecular Reactions. Preprint № 137. Berlin: WIAS, 1994.

[6] Jackson L. K. Subfunctions and second-order ordinary differential inequalities // Adv. Math. 1968. V. 2. P. 308-363.

[7] Бутузов В. Ф., Нестеров А. В. О некоторых сингулярно возмущенных задачах с негладкими погранфункциями // Докл. АН СССР. 1982. Т. 263. № 4. С. 786-789.

Московский государственный университет им. М.В. Ломоносова

Поступило

E-mail: nefedov@mt384.phys.msu.su 29.04.96 